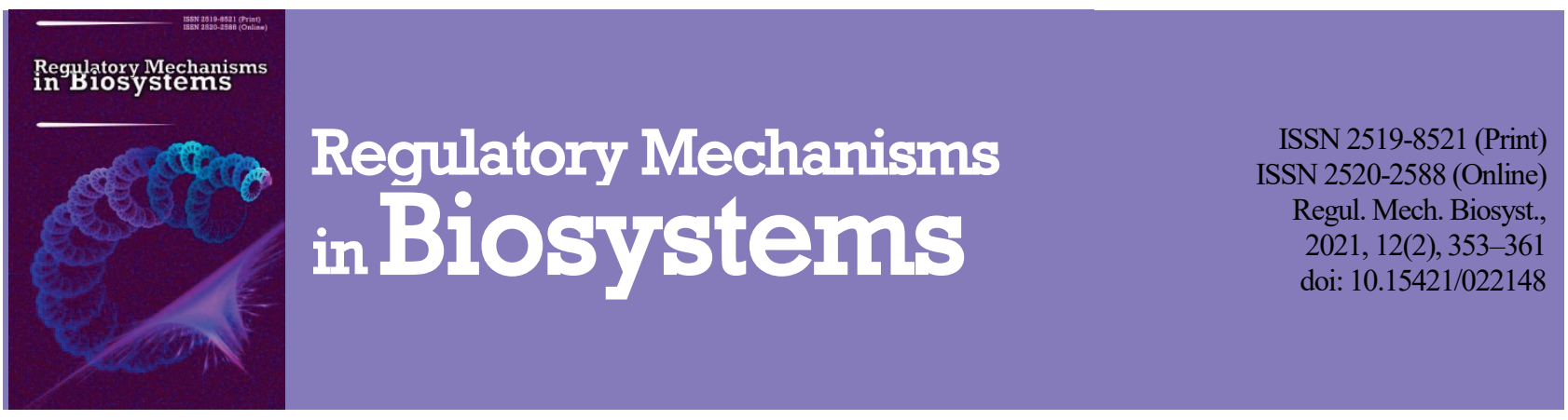

\title{
A systematic review of tapeworms (Plathelminthes, Cestoda) of domestic ducks (Anas platyrhynchos dom.)
}

\author{
F. H. Rzayev*, **, A. M. Nasirov**, E. K. Gasimov* \\ *Azerbaijan Medical University, Baku, Azerbaijan \\ **Institute of Zoology of ANAS, Baku, Azerbaijan
}

Article info

Received 04.05.2021

Received in revised form 09.06.2021

Accepted 10.06.2021

Azerbaijan Medical

University,

Samad Vurgun st., 163 ,

Baku, AZ1078, Azerbaijan

Tel.: +99-412-597-38-98.

E-mail:fuad.zi@mail.ru

Institute of Zoology, ANAS

A. Abbaszadeh st., 1128,

Baku, AZ1004, Azerbaijan

Tel.: +99-412-539-73-71.

E-mail:

fuad.rzayev01f@gmail.com

\begin{abstract}
Rzayev, F. H., Nasirov, A. M., \& Gasimov, E. K. (2021). A systematic review of tapeworms (Plathelminthes, Cestoda) of domestic ducks (Anas platyrhynchos dom.). Regulatory Mechanisms in Biosystems, 12(2), 353-361. doi:10.15421/022148
\end{abstract}

Tapeworms of domestic ducks (Anas platyrhynchos dom.) causing helminthiasis and serious damage to the hosts, cause economic damage in the poultry industry. Helminthological research devoted to the study of the parasite fauna, including tapeworms of domestic ducks, has been carried out in many countries. But there is no review information on the study of the helminth fauna of domestic ducks. We take our own materials and literature data and summarise the information on cestode parasites of Anas platyrhynchos dom., which have been recorded in the world until 2020. Each species is provided with the following data: scientific name, authority and year, intermediate and auxiliary hosts, habitat in the host body, collecting localities and geographic distribution, prevalence and intensity of infection and literature sources. Based on our own research and analysis of literature data, it was revealed that hitherto there are 419 species of helminths (Trematoda - 213, Cestoda - 89, Nematoda - 79, Acanthocephala - 11) recorded parasitising domestic ducks. The tapeworms found in Azerbaijan belong to four families (Dilepididae - 3, Hymenolepididae - 68, Davaineidae - 13, Diphyllobothriidae - 5). In Azerbaijan, 11 out of 89 species of helminths have been found in domestic ducks. Six of them were also noted in the course of our helminthological studies. The cestoda Diorchis inflata was recorded the first time in domestic ducks in Azerbaijan. Most species of cestodes were found in the north-eastern regions of Azerbaijan (Shabran - 11 species, Khachmaz - 6 species) and the southern region (Astara -4 species). All of these areas are located on the shores of the Caspian Sea and are located on the annual migration route of wild birds involved in the spread of cestodes, which are considered biohelminths. Six species (Drepanidotaenia lanceolata, Hymenolepis apodemi, Hymenolepis diminuta, Ligula interrupta, Ligula intestinalis, Schistocephalus solidus) of cestodes parasitize both birds and mammals (D. lanceolata, H. diminuta in humans).

Keywords: cestodes; domestic duck; fauna; systematic classification.

\section{Introduction}

Class Cestoda are endoparasitic tapeworms that spend their adult phase in the digestive system of their final hosts. Tapeworms of domestic ducks (Anas platyrhynchos dom.) causing helminthiasis and serious damage to the hosts, cause economic damage in the poultry industry. Helminthological studies devoted to the study of the parasite fauna, including tapeworms of domestic ducks, have been carried out in many countries (Czaplinski, 1956; Lapage, 1961; Spasskaya, 1966; McDonald, 1969; Smogorzhevskaya, 1976), as well as in Azerbaijan (Shakhtakhtinskaya, 1952, 1959; Shirinov, 1961; Vahidova, 1978; Vahidova et al., 1982). We have studied the species composition, ultrastructure of helminths parasitizing in domestic ducks in our country and developed disease control measures of birds in the last 10 years (Rzayev, 2013; Rzayev \& Ibrahimova, 2015; Seyidbeyli \& Rzayev, 2018; Seyidbeyli \& Maharramov, 2018; Rzayev et al., 2020; Seyidbeyli et al., 2020). It should be noted that, along with the above, in general, there is no review information on the study of the helminth fauna of domestic ducks. There are only articles for some regions. Taking into account the relevance of the topic, on the basis of our own materials and extensive literature, the aim of the present review is to summarise the information on cestode parasites of Anas platyrhynchos dom., which have been recorded in the world until 2020.

The cestoda list is arranged follow the nomenclature and classification by Khalil et al. (2013), Olson et al. (2001), with updates by Kuchta et al. (2008) and Caira et al. (2014). We also took into account the classification adopted by the database of Fauna Europa (www.faunaeur.org). Each species is provided with the following data: scientific name, authority and year, intermediate and auxiliary hosts, habitat in the host body, collecting localities and geographic distribution, prevalence (PI) and intensity (II) of infection and literature sources.

Phylum: Platyhelminthes Gegenbaur, 1859

Class: Cestoda Rudolphi, 1808

Order: Cyclophyllidea Braun, 1900

Family: Dilepididae Fuhrmann, 1907

Genus: Amoebotaenia Cohn, 1899

Amoebotaenia cuneata Linstow, 1872

Intermediate hosts: earthworms

Habitat: small intestine

Distribution: Cosmopolitan. Russia - Moscow region (Petrochenko \& Kotelnikov, 1976) and Egypt - Beni-Suef province (PL 1.18\%, II $3-$ 24 ind.) (El-Dakhly et al., 2020).

Genus: Choanotaenia Railliet, 1896

Choanotaenia infundibulum Bloch, 1779

Intermediate hosts: insects

Habitat: small intestine-jejunum

Distribution: Cosmopolitan. Egypt - Beni-Suef province (PL 0.59\%,

II 13 ind.) (El-Dakhly et al., 2020; McDonald, 1969; Lapage, 1961).

Genus: Platyscolex Spasskaya, 1962

Platyscolex ciliata (Fuhrmann, 1913) Spasskaya, 1962

Intermediate hosts: Simocephalus exspinosus, Daphnia pulex, Moina 
rectirostris (Smogorzhevskaya, 1976).

Habitat: small intestine

Distribution: Holarctic. Bulgaria - northeastern part and Danube river (Marinova et al., 2013), USA and Mexico (PL 5.4\%, II 1-4 ind.) (Farias \& Canaris, 1986), Poland (Lapage, 1961), Kazakhstan, Georgia and Ukraine (PL 0.9\%, II 1-320 ind.) (Ryzhikov, 1967; Smogorzhevskaya, 1976), India (Moghe, 1933).

Family: Hymenolepididae Ariola, 1899

Genus: Aploparaksis Clerc, 1903

Aploparaksis furcigera Rudolphi, 1819

Intermediate hosts: Lumbriculus variegates, Limnodrilus sp.

Habitat: small intestine, large intestine, ceca

Distribution: Holarctic. Russia - Yakutia, Kamchatka (Petrochenko \& Kotelnikov, 1976; Ryzhikov, 1967; Spasskaya, 1966; Ryzhikov et al., 1974; Tolkacheva, 1971; Spassky, 1965), Slovakia (Hanzelova et al., 1995), Poland (Lapage, 1961; Czaplinski, 1956) and UK (PL 2.8\%, II 19 ind.) (Owen, 1951).

Aploparaksis filum Goeze, 1782

Intermediate hosts: Limnodrilus newaensis

Habitat: small intestine, ceca

Distribution: Holarctic and Afrotropic. UK - Wales (Davtes, 1940; Lapage, 1961).

Genus: Cladogynia Baer, 1938

Cladogynia phoeniconaiadis Hudson, 1934

Intermediate hosts: unknown

Habitat: intestine

Distribution: Africa. Egypt - Damanhur city (PL 3.63\%) (Abou Laila et al., 2011).

Genus: Cloacotaenia Wolffhügel, 1938

Cloacotaenia megalops Nitzsch, 1829

Intermediate hosts: Cypris pubera

Habitat: cloaca, distal end of large intestine

Distribution: Cosmopolitan. Russia - Novosibirsk, Omsk, Chelyabinsk, Kurgan regions; Kazakhstan, Uzbekistan, Ukraine (Petrochenko, Kotelnikov, 1976; Ryzhikov, 1967; Spasskaya, 1966), Bulgaria - around the River Danube (Marinova et al., 2013), USA and Mexico (PL 4.6\%, II 1-3 ind.) (Farias \& Canaris, 1986), Egypt (Alexander \& McLaughlin, 1997), Poland (Bezubik, 1956; Lapage, 1961), Germany (Fuhrmann, 1908), North America (Ransom, 1909). Azerbaijan - Shabran region (PL $0.9 \%$, II 6-18 ind.) (Shirinov, 1961). We also reported it in Shabran city (PL 0.24\%, II 1-2 ind.).

\section{Genus: Dicranotaenia Railliet, 1892}

Dicranotaenia coronula (Dujardin, 1845) Railliet, 1892 Intermediate hosts: Ostracoda

Auxiliary hosts: molluscs - Lymnaea ovata, L. palustris, L. vulgaris Habitat: small intestine - tends to occur posteriorly, ceca

Distribution: Holarctic, Neotropical and Australian Regions. Russia Krasnoyarsk, Amur, Rostov regions, Siberia (Spassky, 1963; Spasskaya, 1966; Ryzhikov, 1967; Ryzhikov et al., 1974; Petrochenko \& Kotelnikov, 1976), Ukraine - Dnepropetrovsk, Odessa, Nikolayevsk, Kiev, Khmelnichky, Volyn regions, areas around the Black Sea (PL 3.9\%, II 1552 ind.) (Smogorzhevskaya, 1976), Belarus (Kukar, 2012), Czech Republic and Slovakia (Barus et al., 1977; Hanzelova et al., 1995), Bulgaria Danube and Rositsa rivers (Marinova et al., 2013), Bangladesh - Netrokona and Mymensingh districts (PL $66.7 \pm 22.5 \%$, II 12-110 ind.) (Islam, 1988; Anisuzzaman et al., 2005; Farjana et al., 2008; Yousuf et al., 2009), UK (PL 5.9\%, II 4-19 ind.) (Owen, 1951; Soliman, 1955), Poland (Czaplinski, 1956), Germany (Fuhrmann, 1908), North America (Ransom, 1909). Azerbaijan - Khachmaz and Shabran regions (PL 3.6\%, II 111 ind.) (Shakhtakhtinskaya, 1952, 1959; Shirinov, 1961; Vahidova, 1978; Vahidova et al., 1982).

Genus: Diorchis Clerc, 1903

Diorchis bulbodes Mayhew, 1929

Intermediate hosts: unknown
Habitat: small intestine

Distribution: Europe, Asia, North America. Ukraine - Nikolayev region (PL 0.02\%, II 1 ind.) (Smogorzhevskaya, 1976), Russia - Omsk region (Spasskaya, 1966; Ryzhikov, 1967; Ryzhikov et al., 1974), Mexico and USA (PL 6.2\%, II 1-10 ind.) (Farias \& Canaris, 1986).

Diorchis donis Ajinov, 1960

Intermediate hosts: unknown

Habitat: intestine

Distribution: Europe. Russia - Rostov region (Spassky, 1963; Spasskaya, 1966).

Diorchis elisae (Skrjabin, 1914) Spassky et Freze, 1961

Intermediate hosts: Cypridopsis vidua, Cyclocypris leavis, Diaptomus vulgaris, Dolerocypris fasciata, Notodromas onacha, Eucypris virens (Spasskaya, 1966).

Habitat: small intestine

Distribution: Palaearctic. Russia (Spasskaya, 1966), Belarus (Petrochenko \& Kotelnikov, 1976), Ukraine - Odessa, Nikolayev, Kiev, Khmelnitsky regions, Upper Dniester basin (PL 0.6\%, II 1-126 ind.) (Smogorzhevskaya, 1976), Bulgaria - Nova Zagora, Stara Zagora city (Marinova et al., 2013), Poland (Czaplinski, 1956).

Diorchis formosensis Sugimoto, 1934

Intermediate hosts: unknown

Habitat: ceca, small intestine-duodenum

Distribution: China-Taiwan island (Sugimoto,1934; Schmelz, 1941).

Diorchis inflata (Rudolphi, 1819) Clerc, 1903

Intermediate hosts: Cypridopsis vidua, Cyclocypris laevis, Diantomus vulgaris.

Habitat: small intestine

Distribution: Slovakia (Hanzelova et al., 1995), Ukraine (Smogorzhevskaya, 1976), Russia (Ryzhikov, 1967). We reported this species for the first time in Azerbaijan - Shabran region Shabran city and Bilasuvar region Guneshli village (PL 0.83\%, II 6-25 ind.).

Diorchis longiovum Schiller, 1953

Intermediate hosts: unknown

Habitat: small intestine

Distribution: Bangladesh - Netrokona and Mymensingh regions (PL 23.9\%, II 3-64 ind.) (Farjana et al., 2008; Anisuzzaman et al., 2005).

Diorchis markewitschi Pastschenko, 1952

Intermediate hosts: unknown

Habitat: small intestine.

Distribution: Europe. Ukraine - Kiev region (PL 0.01\%, II 2 ind.)

(Petrochenko \& Kotelnikov, 1976; Smogorzhevskaya, 1976).

Diorchis nyrocae Yamaguti, 1935

Intermediate hosts: Cypridopsis vidua, Cyclocypris laevis (Ostracoda) Habitat: ceca, small intestine - middle region, large intestine

Distribution: Slovakia (Hanzelova et al., 1995).

Diorchis ransomi Schultz, 1940

Intermediate hosts: Cyclocypris laevis, C. ovum, Cypridopsis vidua, Dolerocypris fasciata, Notodromas monacha, Diaptomus caeruleus

Habitat: small intestine- middle region

Distribution: Palaearctic. Ukraine (PL 0.05\%, II 1-13 ind.) (Smogorzhevskaya, 1976), Russia (Spassky, 1963; Spasskaya, 1966; Ryzhikov, 1967).

Diorchis spinata Mayhew, 1929

Intermediate hosts: unknown

Habitat: intestine

Distribution: Holarctic. Poland - River Danube, Dobrich (Marinova et al., 2013).

Diorchis sobolevi Spasskaya, 1950

Intermediate hosts: unknown

Habitat: small intestine 1966).

Distribution: Ukraine - Kiev region (Ryzhikov, 1967; Spasskaya,

Diorchis stefanskii Czaplinski, 1956 
Intermediate hosts: Cypridopsis vidua, C. ovum, Macrocyclops fuscus, Dolerocypris fasciata, Notodromas monacha

Habitat: small intestine-posterior region, large intestine

Distribution: Holarctic. Ukraine (PL 2.2\%, II 1-160 ind.) (Smogorzhevskaya, 1976), Bulgaria (Marinova et al., 2013), Slovakia (Hanzelova et al., 1995), Poland (Czaplinski, 1956), Kazakhstan (Ryzhikov, 1967)

Genus: Diploposthe Jacobi, 1896

Diploposthe laevis Bloch, 1782

Intermediate hosts: Acanthocyclops viridis, Macrocyclops albidus, M. fuscus, Mesocyclops leuckartii, Cypridopsis vidua, Heterocypris incongruens

Habitat: small intestine

Distribution: Cosmopolitan. Bulgaria (Marinova et al., 2013), Poland (Bezubik, 1956; Lapage, 1961), North America (Ransom, 1909), Ukraine (PL 0.07\%, II 2-9 ind.) (Smogorzhevskaya, 1976).

Genus: Echinocotyle Blanchard, 1891

Echinocotyle anatina Krabbe, 1869

Intermediate hosts: Cyprinotus incongruens, Cypris ophthalmica, C. pubera

Habitat: small intestine - posterior portion, large intestine

Distribution: Europe, Asia, Africa, North America. Russia - Rostov, Moscow, Novosibirsk and Saint Petersburg (Ryzhikov, 1967; Petrochenko \& Kotelnikov, 1976), Georgia (Petrochenko \& Kotelnikov, 1976), UK (Soliman, 1955), Poland (Czaplinski, 1956), North America (Ransom, 1909), China - Taiwan island (Sugimoto, 1934; Yamaguti \& Mitunaga, 1943), Ukraine - Kiev, Lvov, Odessa, Donetsk (PL 1.2\%, II 1-17 ind.) (Petrochenko \& Kotelnikov, 1976; Smogorzhevskaya, 1976).

Echinocotyle echinocotyle Fuhrmann, 1907

Intermediate hosts: unknown

Habitat: small intestine - tends to occur in anterior half, ceca

Distribution: Europe (McDonald, 1969)

Echinocotyle rosseteri Blanchard, 1891

Intermediate hosts: Cyclocypris cinerea, C. dispersa, C. globosa, Cypris opthalmica.

Auxiliary hosts: molluscs - Lymnaea ovata, L. ampla, L. peregra, L. stagnalis

Habitat: small intestine - anterior region

Distribution: Holarctic, Indomalaya. Mexico and North America (Farias \& Canaris, 1986; Ransom, 1909), Poland (Czaplinski, 1956).

Genus: Echinolepis Spasskii \& Spasskaya, 1954

Echinolepis carioca Magalhaes, 1898

Intermediate hosts: insects

Habitat: small intestine

Distribution: Egypt - Beni-Suef province, Behera regions (Abou Laila et al., 2011; El-Dakhly et al., 2020), India - Assam region (PL 35.3\%) (Borah et al., 2018), Nigeria - Gombe region (PL 1.3\%) (Adang et al., 2014).

\section{Genus: Drepanidotaenia Railliet, 1892}

Drepanidotaenia lanceolata (Bloch, 1782) Railliet, 1892

Intermediate hosts: crustacean

Auxiliary hosts: mollusc - Lymnaea auricularia

Habitat: small intestine - posterior half

Distribution: Cosmopolitan. Belarus (Kukar, 2012), Czech Republic and Slovakia (Barus et al., 1977; Hanzelova et al., 1995), Bulgaria (Marinova et al., 2013), Bangladesh (Anisuzzaman et al., 2005; Farjana et al., 2008; Aleya et al., 2019), USA and Mexico (Farias \& Canaris, 1986), India - Assam region (Borah et al., 2018), Poland (Czaplinski, 1956). Azerbaijan - Guba, Khachmaz, Astara, Shabran, Zagatala, Masally, Ujar and Yevlakh (PL 47\%, II 2-14 ind.) (Shakhtakhtinskaya, 1952, 1959; Shirinov, 1961; Vahidova, 1978; Vahidova et al., 1982) and Bilasuvar region Chinarly village (PL 16.6\%, II 2-3 ind.) (Aghayeva, 2018). We also reported this parasite in Azerbaijan - Nakhchivan AR in Kengerli region (PL 1.14\%, II 1-2 ind.).

Drepanidotaenia przewalskii (Skrjabin, 1914) Lopep-Neyra, 1942
Intermediate hosts: Eucyclops serrulatus, Mesocyclops crassus, M. leuckarti.

Auxiliary hosts: mollusc-Lymnaea auricularis

Habitat: small intestine - anterior part of duodenum

Distribution: Russia - Bashkortostan (Spassky, 1963; Spasskaya, 1966), Belarus (Kukar, 2012).

Genus: Fimbriaria Froelich, 1802

Fimbriaria amurensis Kotelnikov, 1960

Intermediate hosts: Eucyclops serrulatus, Mesocyclops crassus, M. leuckarti, Diaptomus sarsi

Habitat: small intestine

Distribution: Russia - Khabarvsk and Amur basin (Spasskaya, 1966; Ryzhikov, 1967; McDonald, 1969), Kazakhstan (Petrochenko \& Kotelnikov, 1976).

\section{Fimbriaria fasciolaris Pallas, 1781 (Şək. 4.3)}

Intermediate hosts: crustacean

Habitat: small intestine - anterior region

Distribution: Cosmopolitan. Russia - Bryansk, Kursk, Krasnoyarsk, Amur and Yaroslavl regions (Spasskaya, 1966; Ryzhikov, 1967; Ryzhikov et al., 1974), Tajikistan (PL 11.8\%, II 1-43 ind.) (Borgarenko, 1981), Bulgaria - Rositsa River and Sofia (Marinova et al., 2013), Bangladesh (PL 16.2\%, II 443 ind.) (Islam, 1988; Anisuzzaman et al., 2005; Farjana et al., 2008; Yousuf et al., 2009), USA and Mexico (PL 25.6\%, II 120 ind.) (Farias \& Canaris, 1986), India (PL 22,4\%) (Borah et al., 2018), UK (Wales) (Owen, 1951). Azerbaijan - Shabran (PL 1.1\%, II 1-2 ind.) (Shirinov, 1961). We also reported in Azerbaijan - Shabran (Qala-Alty village), Lankaran, Khachmaz regions (PL 6.5\%, II 1-15 ind.), Nakhchivan $\mathrm{AR}$ - Babek region (PL 4.6\%, II 1-2 ind.).

Fimbriaria kubanika Kotelnikov, 1965

Intermediate hosts: Arctodiaptomus dentifer, Diaptomus mirus, Mesocyclops crassus

Habitat: small intestine

Distribution: Ukraine - Chernigovsk, Zaparozhye, Dnepropetrovsk regions (Smogorzhevskaya, 1976), North Caucasus (Ryzhikov, 1967; Petrochenko \& Kotelnikov, 1976)

Genus: Fimbriarioides Fuhrmann, 1932

Fimbriarioides intermedia Fuhrmann, 1913

Intermediate hosts: Acanthocyclops viridis, Eucyclops serrulatus, Macrocyclops albidus

Habitat: intestine

Distribution: Bangladesh (Anisuzzaman et al., 2005), Ukraine (PL 0.03\%, II 3-15 ind.) (Ryzhikov, 1967; Smogorzhevskaya, 1976).

Genus: Gastrotaenia Wolffhügel, 1938

Gastrotaenia dogieli Gynezynskaja, 1944

Intermediate hosts: Diatomus sp., Arctodiaptomus bacillifer, Arctodiaptomus calinus

Habitat: gizzard-under lining

Distribution: Palaearctic. Kazakhstan, Russia - Southern Urals, Western Siberia (Ryzhikov, 1967; Petrochenko \& Kotelnikov, 1976).

Genus: Hymenolepis Weinland, 1858

Hymenolepis angularostris Sugimoto, 1934

Intermediate hosts: unknown

Habitat: intestine

Distribution: China - Taiwan island (Sugimoto, 1934; McDonald, 1969).

Hymenolepis apodemi Makarikov, 2013

Intermediate hosts: unknown

Habitat: small intestine

Distribution: Egypt - Beni-Suef province (PL 0.78\%, II 6-24 ind.)

(El-Dakhly et al., 2020).

Hymenolepis cantaniana Polonio, 1860

Intermediate hosts: insects - Ataenius cognatus, A. stercorator, Choeridium histeroides

Habitat: small intestine 
Distribution: Nigeria - Gombe city (Paul et al., 2015).

Hymenolepis diminuta Rudolphi, 1819

Intermediate hosts: Arthrapoda

Habitat: faeces, small intestine

Distribution: Cosmopolitan. Bangladesh - Sonarqaon province (Aleya et al., 2019).

Hymenolepis infrequens Sharma, 1943

Intermediate hosts: unknown

Habitat: small intestine

Distribution. Asia. Myanmar (Sharma, 1943; McDonald, 1969).

Hymenolepis sagitta (Rosseter 1906) Fuhrmann 1908

Intermediate hosts: unknown

Habitat: intestine

Distribution: Poland (Czaplinski, 1956), North America (Ransom, 1909).

Hymenolepis tenerrima (Linstow 1882) Fuhrmann 1906

Intermediate hosts: Herpetocypris reptans

Habitat: large intestine

Distribution: North America (Ransom, 1909; McDonald, 1969).

Genus: Lobatolepis Yamaguti, 1959

Lobatolepis lobulata Mayhew, 1925

Intermediate hosts: unknown

Habitat: small intestine

Distribution: Asia. Bangladesh - Mymensingh district (Anisuzzaman et al., 2005).

Genus: Microsomacanthus Lopez-Neyra, 1942

Microsomacanthus abortiva (Linstow, 1904) Lopez-Neyra, 1942

Intermediate hosts: Gammarus pulex

Habitat: ceca, small intestine

Distribution: Bulgaria - İskar, Yantra rivers (Marinova et al, 2013), Ukraine (PL 0.1\%, II 1-109 ind.) (Smogorzhevskaya, 1976), UK (PL 18.0\%, II 13-23 ind.) (Soliman, 1955), Africa - Egypt and Nigeria (Alexander \& McLaughlin, 1997), Bangladesh - Mymensingh district (Anisuzzaman et al., 2005).

Microsomacanthus arcuata Kowalewski, 1904

Intermediate hosts: unknown

Habitat: intestine

Distribution: Palaearctic. Bangladesh - Mymensingh district (McDonald, 1969; Anisuzzaman et al., 2005).

Microsomacanthus collaris Batsch, 1786

Intermediate hosts: crustacean

Auxiliary hosts: molluses - Lymnaea palustris, L. vulgaris

Habitat: Small intestine- total length

Distribution: Cosmopolitan. Ukraine (PL 7.7\%, II 1-1342 ind.) (Smogorzhevskaya, 1976), Russia - Far East, Eastern and Western Siberia, along the Volga River (Ryzhikov, 1967; Spasskaya, 1966; Ryzhikov et al., 1974), Tajikistan (PL. 0.95\%, II 3-8 ind.) (Borgarenko, 1981), Georgia (Petrochenko \& Kotelnikov, 1976), Belarus (Kukar, 2012), Bangladesh (PL 64\%, II 2-840 ind.) (Islam, 1988), UK (PL 12.3\%, II 9 13 ind.) (Soliman, 1955), India - Assam province (PL 35.8\%) (Borah et al., 2018), North America (Ransom, 1909), China - Taiwan (Sugimoto, 1934). Azerbaijan - Astara, Shabran, Khachmaz regions (PL 26.6\%, II 4 13 ind.) (Shakhtakhtinskaya, 1952, 1959; Shirinov, 1961; Vahidova, 1978; Vahidova et al., 1982).

\section{Microsomacanthus compressa Linton, 1892}

Intermediate hosts: Cyclops strenuus, Macrocyclops albidus, Mesocyclops leuckarti

Auxiliary hosts: molluscs - Anisus spirorbis, Lymnaea corneus, L. ovata, L. palustris, L. peregra, L. stagnalis, Planorbis planorbis, Valvata cristata, Viviparus viviparus

Habitat: small intestine - total length

Distribution: Holarctic. Russia (Spasskaya, 1966; Ryzhikov, 1967; Petrochenko \& Kotelnikov, 1976), Tajikistan (PL 1.42\%, II 7-35 ind.) (Borgarenko, 1981), Kazakhstan (Egizbaeva, 1971), Uzbekistan (Sultanov, 1963), Belarus (Kukar, 2012), Bulgaria - River Danube (Marinova et al., 2013), Poland (Bezubik, 1956; Czaplinski, 1956). Azerbaijan Astara, Shabran, Gusar, Salyan, Khachmaz, Yevlakh, Ujar, Masally, Lankaran regions (PL 42.6\%, II 1-111 ind.) (Shirinov, 1961; Vahidova, 1978).

Microsomacanthus fausti Tseng-Shen, 1932

Intermediate hosts: Acanthocyclops viridis, Mesocyclops crassus, Mesocyclops leuckarti, Mesocyclops oithonoides

Auxiliary hosts: molluscs - Planorbidae

Habitat: small intestine, ceca

Distribution: Palaearctic. Bulgaria (Marinova et al., 2013), Ukraine Zaporozhye region (PL 0.2\%, II 1-72 ind.) (Smogorzhevskaya, 1976), Russia (Ryzhikov, 1967).

Microsomacanthus jamunicus (Sharma, 1943) Yamaguti, 1959

Intermediate hosts: unknown

Habitat: small intestine

Distribution: Asia. Myanmar (Sharma, 1943; McDonald, 1969).

Microsomacanthus microsoma Creplin, 1829

Intermediate hosts: Eucyclops agilis, Eucyclops serrulatus, Gammarus locusta

Auxiliary hosts: molluscs - Lymnaea lagotus, L. peregra, Radix logotus, L. stagnalis

Habitat: small intestine

Distribution: Russia - Western Siberia, Far East (Spasskaya, 1966; Ryzhikov, 1967; Petrochenko \& Kotelnikov, 1976), Belarus (Ryzhikov, 1967), Tajikistan (PL 4.74\%, II 5-32 ind.) (Borgarenko, 1981), Uzbekistan-Tashkent and Bukhara provinces (Sultanov, 1963).

Microsomacanthus oidemiae Spassky et Jurpalova, 1964

Intermediate hosts: unknown

Habitat: small intestine

Distribution: Tajikistan - Pyandjinsk region (PL 0.47\%, II 5 ind.) (Borgarenko, 1981)

Microsomacanthus pachycephala von Linstow, 1872

Intermediate hosts: unknown

Habitat: Small intestine

Distribution: Russia-Chelyabinsk (Ryzhikov, 1967).

Microsomacanthus paracompressa Czaplinski, 1956

Intermediate hosts: Acanthocyclops bicuspidatus, A. viridis, Cyclops gigas, Eucyclops macruroides, E. serrulatus, Mesocyclops crassus, M. leuckarti, M. oithonoides

Auxiliary hosts: molluscs - Acroloxus lacustris, A. palustris, Amphipepla gluteosa, Lymnea ampla, L. auricularis, L. ovata, L. palustris, L. stagnalis, Planorbis planorbis

Habitat: small intestine

Distribution: Palaearctic. Belarus (Kukar, 2012), Bulgaria - River Danube and northern shores of the Black Sea (Marinova et al., 2013), Poland (Czaplinski, 1956; Shirinov, 1961), Ukraine - shores of the Black Sea (PL 0.5\%, II 2-123 ind.) (Smogorzhevskaya, 1976), Tajikistan Kanibadam poultry (PL 1.4\%, II 4-22 ind.) (Borgarenko, 1981), Kazakhstan - Pavlodar (Egizbaeva, 1971). Azerbaijan - Shabran (PL 0.9\%, II 4-16 ind.) (Shirinov, 1961; Vahidova, 1978).

Microsomacanthus paramicrosoma Gasowska, 1931

Intermediate hosts: Acanthocyclops bicuspidatus, A. viridis, Eucyclops serrulatus, Macrocyclops albidus, Mesocyclops crassus, M. leuckarti, M. oithonoides

Auxiliary hosts: molluscs - Galba palustris, Planorbis planorbis, Lymnaea auricularia, L. ovata, L. palustris, L. peregra, L. stagnalis

Habitat: small intestine - anterior and midregion

Distribution: Holarctic. Bulgaria - Danube river and surrounding swamps (Marinova et al., 2013), Poland (Czaplinski, 1956), Ukraine (PL 0.9\%, II 4-3010 ind.) (Smogorzhevskaya, 1976), Russia - Khabarovsk, Amur regions (Ryzhikov et al., 1974; Shirinov, 1961), Tajikistan - Kanibadamsk Poultry (PL 3.3\%, II 5-12 ind.) (Borgarenko, 1981), KazakhstanPavlodar, Almaty (Egizbaeva, 1971). Azerbaijan - Shabran (port of Devechi) and Astara regions (PL 6.5\%, II 4-27 ind.) (Vahidova, 1978; Vahidova et al., 1982). We also reported in Azerbaijan - Shabran region (Gala Alty village) (PL 4.35\%, II 5-8 ind.). 
Microsomacanthus parvula Kowalewski, 1904

Intermediate hosts: Hirudinea - Erpobdella atomaria, E. octoculata, E. punctata

Habitat: small intestine - generally in anterior half

Distribution: Holarctic. Bulgaria - Vratsa city (Marinova et al., 2013), Poland (Czaplinski, 1956), Germany (Fuhrmann, 1908), North America (Ransom, 1909), Japan (Uchida, 1991), Ukraine - Kharkiv, Odessa and Nikolaev regions (PL 1.2\%, II 2-40 ind.) (Petrochenko \& Kotelnikov, 1976), Russia (Ryzhikov, 1967).

Microsomacanthus rangdonensis Spasskiy, Dang Van-Ngy \& Fürpalova, 1963

Intermediate hosts: unknown

Habitat: small intestine

Distribution: Asia. Vietnam (McDonald, 1969).

Microsomacanthus spiralibursata Czaplinski, 1956

Intermediate hosts: Acanthocyclops viridis, Macrocyclops albidus, Mesocyclops leuckarti

Auxiliary hosts: molluscs - Lymnaea ovata, L. auricularia, L. stagnalis.

Habitat: small intestine - entire length

Distribution: Kazakhstan (Ryzhikov, 1967).

Microsomacanthus spiralicirrata Maksimova, 1963

Intermediate hosts: unknown

Habitat: small intestine

Distribution: Kazakhstan - Aktobe region and shores of the Black

Sea (Spasskaya, 1966; McDonald, 1969).

Microsomacanthus trichorhynchus Yoshida, 1910

Intermediate hosts: unknown

Habitat: small intestine-duodenum

Distribution: Russia - Rostov region and Japan (Spasskaya, 1966; McDonald, 1969).

Genus: Retinometra Spasskii, 1955

Retinometra giranensis Sugimoto, 1934

Intermediate hosts: unknown

Habitat: small intestine

Distribution: Palaearctic. Russia - Rybinsk Reservoir and Tuva (Spasskaya, 1966).

Retinometra longicirrosa (Fuhrmann, 1906) Spassky, 1963

Intermediate hosts: Acanthocyclops viridis, Cyclops strenuus, Eucyclops agilis, Eucyclops serrulatus, Mesocyclops leuckarti, Diaptomus coeruleus, Diaptomus sarsi

Habitat: small intestine

Distribution: Palaearctic, Indomalaya. Russia - Kalijsk, Kursk, Bryansk, Tula, Kursk, Yaroslavl and Ryazan, Omsk, Chelyabinsk regions, Bashkortostan, Western Siberia, mouth of the Volga River (Petrochenko \& Kotelnikov, 1976; Ryzhikov et al., 1974), India (Southwell, 1930), France (Joyeux \& Baer, 1936).

Retinometra meggitti Sharma, 1913

Intermediate hosts: unknown

Habitat: small intestine

Distribution: Asia. Myanmar (McDonald, 1969).

Retinometra oshimai (Sugimoto, 1934) Spasskii, 1963

Intermediate hosts: unknown

Habitat: small intestine

Distribution: Asia. China-Taiwan island (McDonald, 1969).

Retinometra pittalugai Lopez-Neyra, 1932

Intermediate hosts: unknown

Habitat: small intestine

Distribution: Spain and Russia (Spasskaya, 1966; McDonald, 1969).

Retinometra rangoonica (Sharma, 1943) Spasskii, 1963

Intermediate hosts: unknown

Habitat: small intestine

Distribution: Asia. Myanmar (McDonald, 1969).

Retinometra venusta (Rosseter, 1897)

Intermediate hosts: Cyclocypris dispersa, C. laevis, Notodromas mo- nacha, Acanthocyclops viridis, Eucyclops macruroides, Macrocyclops albidus, M. fuscus

Habitat: small intestine - anterior region.

Distribution: Russia and North America (Ransom, 1909; Gower, 1939; Spasskaya, 1966).

Genus: Sobolevicanthus Spasskii \& Spasskaya, 1954

Sobolevicanthus columbae Zeder, 1800

Intermediate hosts: unknown

Habitat: small intestine

Distribution: Bangladesh - Sonargaon Upazila and Narayanganj (Aleya et al., 2019).

Sobolevicanthus gracilis Zeder, 1803

Intermediate hosts: crustacean

Auxiliary hosts: molluscs - Lymnaea palustris, L. vulgaris

Habitat: small intestine - entire length.

Distribution: Holarctic, Indomalaya. Ukraine - Dnipropetrovsk, Odessa, Nikolayevsk, Kiev, Khmelnitsky, Volynsk (PL 5.9\%, II 1536 ind.) (Smogorzhevskaya, 1976), Bulgaria - River Rositsa (Marinova et al., 2013), USA and Mexico (Farias \& Canaris, 1986), UK (Owen, 1951; Soliman, 1955), Poland (Czaplinski, 1956), France (Joyeux \& Baeb, 1936), India (Southwell, 1930), China - Taiwan island (Sugimoto, 1934), Tajikistan - Pyandjinsk region (PL 10.4\%, II 5-18 ind.) (Borgarenko, 1981), Russia - Krasnoyarsk, Amur (Ryzhikov, 1967; Ryzhikov et al., 1974). Azerbaijan - Shabran region (PL 1.8\%, II 6-58 ind.) (Shirinov, 1961; Vahidova, 1978; Vahidova et al., 1982).

Sobolevicanthus krabbeella Hughes, 1940

Intermediate hosts: crustacean

Habitat: small intestine, ceca

Distribution: Palaearctic. Russia - Southern Urals (Ryzhikov, 1967; McDonald, 1969).

Sobolevicanthus octacanthus Krabbe, 1869

Intermediate hosts: Cyclops vicinus, Eucyclops serrulatus, Mesocyclops oithonoides.

Habitat: small intestine - posterior region more generally

Distribution: Palaearctic. Ukraine - Odessa, Nikolayev regions and Black Sea coast (PL 0.4\%, II 4-16 ind.) (Smogorzhevskaya, 1976), Russia (Ryzhikov, 1967).

Genus: Tschertkovilepis Spasskii \& Spasskaya, 1954

Tschertkovilepis krabbei Kowalewski, 1895

Intermediate hosts: crustacean

Auxiliary hosts: mollusc-Lymnaea auricularia

Habitat: small intestine

Distribution: Holarctic. Ukraine (Spasskaya, 1966; Smogorzhevskaya, 1976; Yuskiv \& Melnychuk, 2020), Russia - Braynsk, Omsk, Amur, Kursk, Yaroslavl, Novosibirsk, Orlov, Saint Petersburg, Rybinsk Reservoir, Tatarstan (Spasskaya, 1966; Ryzhikov et al., 1974), Bulgaria (Marinova et al., 2013), France (Joyeux \& Baeb, 1936), Poland (Bezubik, 1956; Czaplinski, 1956).

Tschertkovilepis setigera Frölich, 1789

Intermediate hosts: Acanthocyclops bicuspidatus, Cyclops gigas, C. strenuus, Eucyclops serrulatus, E. speratus, Mesocyclops leuckarti, M. oithonoides, Diaptomus coeruleus, D. sarsi.

Auxiliary hosts: molluses - Galba palustris, Lymnaea auricularia, L. ovata, L. palustris, L. stagnalis, Planorbarius corneus, Coretus corneus Habitat: small intestine

Distribution: Palaearctic, Indomalaya. Ukraine - Odessa, Nikolayev, Kiev, Poltava regions (PL 1.4\%, II 1-160 ind.) (Smogorzhevskaya, 1976; Yevstafyeva \& Yeresko, 2018; Yevstafyeva et al., 2018; Yuskiv \& Melnychuk 2020; Yevstafieva et al., 2020), Russia - Bashkortostan, Dagestan, Kaluga, Kursk, Ryazan, Bryansk, Yaroslavl, Omsk, Novosibirsk, Amur (Ryzhikov et al., 1974), Bulgaria - Pavlikeni, Veliko Tarnovo, Elena regions (Marinova et al., 2013), Poland (Czaplinski, 1956), China - Taiwan (Sugimoto,1934), Tajikistan (PL 0.47\%, II 1 ind.) (Borgarenko, 1981), Uzbekistan (Sultanov, 1963), Turkmenistan (Kibakin, 1966). Azerbaijan Astara, Khachmaz, Shabran, Lankaran regions (PL 17.7 \%, II 1-42 ind.) (Shakhtakhtinskaya, 1952; Shirinov, 1961; Vahidova, 1978; Vahidova 
et al., 1982). We reported in Jalilabad (PL 2.17\%, II 12 ind.) and in Nakhchivan AR - Julfa, Ordubad, Sharur (PL 5.71\%, II 3-7 ind.).

Genus: Wardium Mayhew, 1925

Wardium aequabilis Rudolphi, 1810

Intermediate hosts: Cyclocypris laevis, Macrocyclons fuscus

Habitat: small intestine - posterior portion, ceca

Distribution: Russia (Petrochenko \& Kotelnikov, 1976; Ryzhikov, 1967; Spasskaya, 1966).

Wardium creplini Krabbe, 1869

Intermediate hosts: unknown

Habitat: small intestine

Distribution: Russia - Amur (Ryzhikov et al., 1974).

Family: Davaineidae Braun, 1900

Genus: Baerfainia Yamaguti, 1959

Baerfainia anoplocephaloides Baer \& Fain 1955

Intermediate hosts: unknown

Habitat: intestine 2011).

Distribution: Egypt - Damanhur city (PL 3.63\%) (Abou Laila et al,

Genus: Cotugnia Diamare, 1893

Cotugnia digonopora Pasquale, 1890

Intermediate hosts: insects

Habitat: small intestine

Distribution: Egypt - Beni-Suef province and Bangladesh - Dhaka (Aleya et al., 2019; El-Dakhly et al., 2020).

Cotugnia fastigata Meggitt, 1920

Intermediate hosts: insects

Habitat: intestine

Distribution: Asia. Myanmar (Southwell, 1930).

Genus: Raillietina Fuhrmann, 1920

Raillietina anatina Fuhrmann, 1909

Intermediate hosts: insects

Habitat: intestine

Distribution: North America (Ransom, 1909), Italy (McDonald, 1969).

Raillietina birmanica Meggitt, 1926

Intermediate hosts: insects

Habitat: intestine

Distribution: Asia. Myanmar (McDonald, 1969; Sharma, 1943).

Raillietina bonini Megnin, 1899

Intermediate hosts: insects

Habitat: intestine

Distribution: Bangladesh - Dhaka, Narayanganj (Aleya et al., 2019).

Raillietina cesticillus (Molin, 1858) Railliet, 1921

Intermediate hosts: insects

Habitat: small intestine - duodenum and jejunem

Distribution: Cosmopolitan. Egypt - Beni-Suef province (El-Dakhly et al., 2020), Bangladesh - Dhaka, Narayanganj (Aleya et al., 2019), India-Assam province (Borah et al., 2018).

Raillietina echinobothrida Megnin, 1881

Intermediate hosts: insects

Habitat: small intestine

Distribution: Cosmopolitan. Egypt - Beni-Suef province (El-Dakhly et al., 2020), Bangladesh - Dhaka, Narayanganj (Aleya et al., 2019), Nigeria - Gombe, İlorin (Paul et al., 2015; Ola-Fadunsin et al., 2016), India Upper Assam (Borah et al., 2018), Tanzania (Rukambile et al., 2020).

Raillietina magninumida Jones, 1930

Intermediate hosts: insects

Habitat: small intestine

Distribution: Nigeria - Gombe (Adang et al., 2014).

Raillietina microcotyle Skrjabin, 1914

Intermediate hosts: insects
Habitat: intestine

Distribution: Italy (McDonald, 1969).

Raillietina osakensis Iwata \& Tamura, 1933

Intermediate hosts: insects

Habitat: intestine

Distribution: Japan (Iwata \& Tamura, 1933; McDonald, 1969).

Raillietina parviuncinata Meggitt \& Po Saw, 1921

Intermediate hosts: insects

Habitat: intestine

Distribution: Asia. Myanmar (Southwell, 1930) and China (McDonald, 1969)

Raillietina tetragona (Molin, 1858)

Intermediate hosts: insects

Habitat: cloaca, small intestine

Distribution: Egypt - Beni-Suef province (El-Dakhly et al., 2020), Nigeria - Gombe (Paul et al., 2015), Iran - Gilan province (Bahar et al., 2017), India - Upper Assam (Borah et al., 2018), Tanzania - Morogoro (Muhairwa et al., 2007; Rukambile et al., 2020).

Order: Diphyllobothriidea Kuchta, Scholz, Brabec, Bray, 2008

Family: Diphyllobothriidae Lühe, 1910

Genus: Ligula Bloch, 1782

Ligula colymbi Zeder, 1803

Intermediate hosts: Acanthocyclops bicuspidatus, A. viridis, Cyclops strenuus

Auxiliary hosts: pisces - Barbus brachycephalus, Cobitis taenia, Gobio gobio, Leucaspius delineatus, Nemachilus kuschkewitschi, Nemachilus dorsalis, Nemachilus stoliczkai, N. strauchi.

Habitat: small intestine

Distribution: Ukraine (Ryzhikov, 1967).

Ligula interrupta Rudolphi, 1810

Intermediate hosts: Acanthocyclops bicuspidatus, A. vernalis, $A$. viridis, Cyclops furcifer, C. insignis, C. strenuus, Eucyclops serrulatus, $M a-$ crocyclops albidus, Diaptomus glacialis.

Auxiliary hosts: pisces - Abramis brama, Barbus lacerta, Carassius auratus, C. carassius, Cyprinus carpio, Gobio albipennatus, Leuciscus brandti, Rhodeus sericeus, Rutilus rutilus.

Habitat: intestine

Distribution: Ukraine - Kiev and Crimea (PL 0.04\%, II 1-2 ind.) (Ryzhikov, 1967; Petrochenko \& Kotelnikov, 1976; Smogorzhevskaya, 1976).

Ligula intestinalis (Linnaeus, 1758) Gmelin, 1790

Intermediate hosts: Acanthocyclops bicuspidatus, A. viridis, Cyclops strenuus, Eucyclops serrulatus, Mesocyclops leuckarti, M. oithonoides, Diaptomus glacialis, D. gracilis, D. sarsi.

Auxiliary hosts: Pisces

Habitat: small intestine

Distribution: China - Taiwan (Sugimoto, 1934), Ukraine - Crimea, Dniester (PL 0.2\%, II 1-27 ind.) (Petrochenko \& Kotelnikov, 1976; Smogorzhevskaya, 1976), Russia - Altai (Petrochenko \& Kotelnikov, 1976).

Genus: Schistocephalus Creplin, 1829

Schistocephalus pungitii Dubinina, 1959

Intermediate hosts: Acanthocyclons bicuspidatus, A. gigas, A. viridis, Cyclops furcifer, C. strenuus, Macrocyclops albidus, M. fuscus, Diaptomus gracilis

Auxiliary hosts: fish - Pungitius pungitius

Habitat: small intestine

Distribution: Europe, Asia, North America (McDonald, 1969).

Schistocephalus solidus Müller, 1776

Intermediate hosts: Acanthocyclops bicuspidatus, A. eigas, Cyclops furcifer, C. strenuus, Eucyclops macrurus, E. macruroides, Macrocyclops albidus, M. fuscus, Mesocyclops leuckarti.

Auxiliary hosts: pisces

Habitat: small intestine

Distribution: UK (Crompton \& Nesheim, 1976), Poland (Bezubik, 1956), France (Joyeux \& Baer, 1936), Russia (Ryzhikov, 1967). 


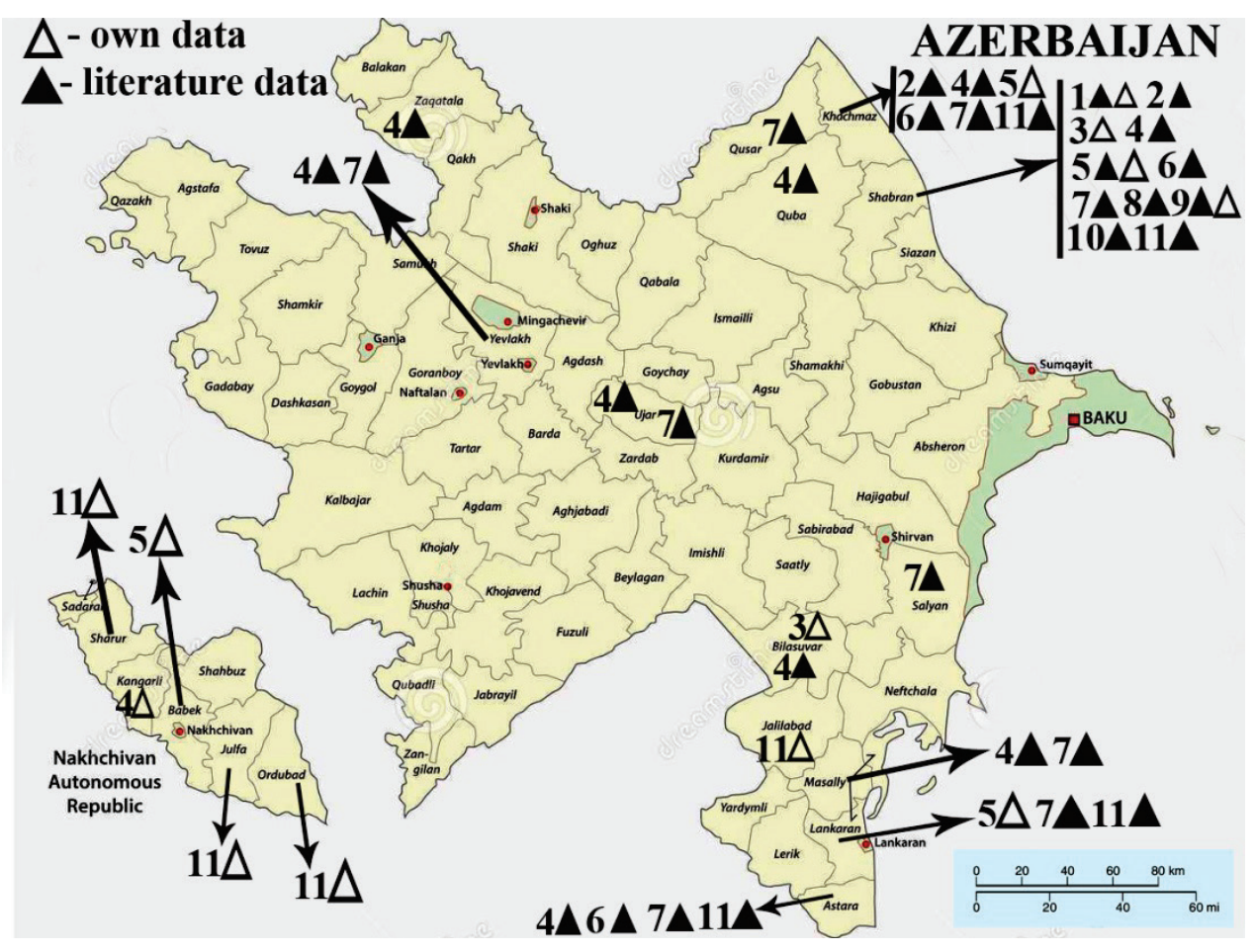

Fig. 1. Map of the areas of occurrence of cestodes in domestic ducks in Azerbaijan: 1-Cloacotaenia megalops, 2-Dicranotaenia coromula, 3-Diorchis inflata, 4-Drepanidotaenia lanceolata, 5-Fimbriaria fasciolaris, 6-Microsomacanthus collaris, 7-Microsomacanthus compressa, 8-Microsomacanthus paracompressa, 9-Microsomacanthus paramicrosoma, 10-Sobolevicanthus gracilis, 11-Tschertkovilepis setigera

\section{Conclusion}

Based on our own research and analysis of literature data, it was revealed that hitherto there are 419 species of helminths (Trematoda -213 , Cestoda - 89, Nematoda - 79, Acanthocephala - 11) in domestic ducks (Anas platyrhynchos dom.). The tapeworms found in Azerbaijan belong to four families (Dilepididae - 3, Hymenolepididae - 68, Davaineidae 13, Diphyllobothriidae - 5). In Azerbaijan, 11 out of 89 species of helminths (C. megalops, D. coronula, D. inflata, D. lanceolata, $F$. fasciolaris, $M$. collaris, $M$. compressa, $M$. paracompressa, $M$. paramicrosoma, $S$. gracilis, T. setigera) are found in domestic ducks (Fig. 1). Six of them $(C$. megalops, $D$. inflata, $D$. lanceolata, $F$. fasciolaris, $M$. paramicrosoma, $T$. setigera) were also noted in the course of our helminthological studies. The cestoda $D$. inflata was recorded for the first time in domestic ducks in Azerbaijan. Most species of cestodes were found in the north-eastern regions of Azerbaijan (Shabran - 11 species, Khachmaz - 6 species) and southern region (Astara -4 species). All of these areas are located on the shores of the Caspian Sea and are located on the annual migration route of wild birds involved in the spread of cestodes, which are considered biohelminths. Six species (Drepanidotaenia lanceolata, Hymenolepis apodemi, H. diminuta, Ligula interrupta, L. intestinalis, Schistocephalus soli$d u s$ ) of cestodes parasitize both birds and mammals (D. lanceolata, $H$. diminuta in humans). D. lanceolata is also recorded in Azerbaijan.

\section{References}

Abou Laila, M., El-Bahy, N., Hilali, M., Yokoyama, N., \& Igarashi, I. (2011). Prevalence of the enteric parasites of ducks from Behera governorate. The Joumal of Protozoology Research, 21, 36-44.

Adang, K. L., Asher, R., \& Abba, R. (2014). Gastrointestinal helminths of domestic chicken Gallus domesticus and ducks Anas platyrhynchos slaughtered at Gombe main markets, Gombe State, Nigeria. Asian Joumal of Poultry Science, $8,32-40$.

Aghayeva, Z. T. (2018). Azerbaycanin muxtelif rayonlarinda gaz (Anser anser dom.) ve ordeklerin (Anas platherhynchos dom.) helmintlerin bio-ekoloji xususiyyetlerinin oyrenilmesi [Study of bio-ecological features of helminths of goose (Anser anser dom.) and duck (Anas platherhynchos dom.) in different regions of Azerbaijan]. Elm, Baku (in Azerbaijanian).
Alexander, S. J., \& McLaughlin, J. D. (1997). A checklist of helminths from the respiratory system and gastrointestinal tracts of African Anatidae. Onderstepoort Journal of Veterinary Research, 64, 5-16.

Aleya, B., Mandira, M., Farjana, A., \& Subrina, S. (2019). Occurrence of parasites in domestic ducks from rural areas of Narayanganj. Bangladesh Joumal of Zoology, 47(2), 315-323.

Anisuzzaman, M. A., Alim, M. H., Rahman, \& Mondal, M. M. H. (2005). Helminth parasites in indigenous ducks: Seasonal dynamics and effects on production performance. Journal of the Bangladesh Agricultural University, 3(2), 283-290.

Bahar, S., Shahrokh, R., \& Mohamad, D. (2017). Prevalence and intensity of parasitic infection in domestic ducks (Anas platyrhynchos) in Gilan Province, Northem Iran. Comparative Clinical Pathology, 26, 165-167.

Barus, V., Micolasek, A., \& Busta, J. (1977). Influence of breeding technology on helminthfauna of geese (Anser anser $\mathrm{f}$. dom.). Folia Parasitologica, 24, 305-314.

Bezubik, B. (1956). Helmintofauna dzikich kaczek (podrodzina Anatinae) woj lubelskiego i białostockiego [Helminthological fauna in wild ducks (subfamily Anatinae) in the Lublin and Bialystok regions]. Wiadomosci Parazytologiczne, 2, 267-268 (in Polish).

Borah, N., Phukan, S. C., Islam, S., Tamuli, S., Tamuli, S. M., \& Rajbongshi, P. (2018). Prevalence of helminth parasites of domestic ducks in Upper Assam (India). International Joumal of Chemical Studies, 6(4), 131-134.

Borgarenko, L. F. (1981). Gel'minty ptits Tadzhikistana. Tsestody [Helminths of birds of Tajikistan. Cestodes]. Donish, Dushanbe (in Russian).

Caira, J. N., Jensen, K., Waeschenbach, A., Olson, P. D., \& Littlewood D. T. J. (2014). Orders out of chaos-molecular phylogenetics reveals the complexity of shark and stingray tapeworm relationships. International Joumal for Parasitology, 44, 55-73.

Crompton, D. W. T., \& Nesheim, M. C. (1976). Host-parasite relationships in the alimentary tract of domestic birds. Advances in Parasitology, 14, 95-194.

Czaplinski, B. (1956). Hymenolepididae Fuhrmann, 1907 (Cestoda). Parasites of some domestic and wild Anseriformes in Poland. Acta Parasitologica Polonica, 4, 175-373.

Davtes, T. I. (1940). Three closely related species of Aploparaksis Clerc, 1903 Parasitology, 32, 198-207.

Egizbaeva, H. I. (1971). Obzor gel'mintofauny domashnikh utok i gusey v Kazakhstane i Sredney Azii [Review of helminthofana of domestic ducks and geese in Kazakhstan and Central Asia]. Proceedings of the Institute of Zoology of the Academy of Sciences of the Kazakh SSR, 31, 60-68 (in Russian).

El-Dakhly, K. M., Mohamed, H. I., Kamel, A. A., Mahrous, L. N., Amany, E. E., \& Aboshinaf, S. M. (2020). Prevalence, distribution pattern and pathological alterations of gastrointestinal helminthosis in domestic ducks in Beni-Suef, Egypt. Joumal of Advanced Veterinary Research, 10(1), 1-8. 
Farias, J. D., \& Canaris, A. G. (1986). Gastrointestinal helminths of the Mexican duck, Anas platyrhynchos diazi Ridgway, from north Central Mexico and Southwestern United States. Joumal of Wildlife Desaeses, 22(1), 51-54.

Farjana, T., Islam, K. R., \& Mondal, M. M. H. (2008). Population density of helminths in ducks: Effects of host's age, sex, breed and season. Bangladesh Journal of Veterinary Medicine, 6(1), 45-51.

Fuhrmann, O. (1908). Die cestoden der vogel [Cestodes of the birds]. Zoological Yearbook, 10, 1-232 (in German).

Gower, W. C. (1939). Host-parasite catalogue of the helminths of ducks. American Midland Naturalist, 22(3), 580-628.

Hanzelova, V., Rysavy, B., \& Snabel, V. (1995). Synopsis of cestodes in Slovakia III. Cyclophyllidea: Amabiliidae, Acoleidae, Catenotaeniidae, Davaineidae, Hymenolepididae. Helminthologia, 32, 67-73.

Islam, M. R., Shaikh, H., \& Baki, M. A. (1988). Prevalence and pathology of helminth parasites in domestic ducks of Bangladesh. Veterinary Parasitology, 29, $73-77$.

Iwata, S., \& Tamura, O. (1933). Some intestinal parasites in the duck from Japan. Annotationes Zoologicae Japonenses, 14(1), 1-6.

Joyeux, C. E., \& Baer, J. G. (1936). Cestodes. Faune de France. Fed. Franc. Soc. Sc. Nat., 30, 1-613.

Khalil, L. F., Jones, A., \& Bray, R. A. (1994). Keys to the cestode parasites of vertebrates. CABI, Wallingford.

Kibakin, V. V. (1966). K izucheniyu gel'mintov ptits Yuzhnykh rayonov Turkmenii [Study of bird helminths in the southem regions of Turkmenistan]. Proceedings of the Turkmenian Academy of Sciences, 5, 85-89 (in Russian).

Kuchta, R., Scholz, T., Brabec, J., \& Bray, R. A. (2008). Suppression of the tapeworm order Pseudophyllidea (Platyhelminthes: Eucestoda) and the proposal of two new orders, Bothriocephalidea and Diphyllobothriidea. International Journal for Parasitology, 38(1), 49-55.

Kukar, D. V. (2012). Osobennosti gel'mintofauny dikikh i domashnikh utok v Severnoy zone Belarusi [Features of the helminth fauna of wild and domestic ducks in the Northern zone of Belarus]. Actual Problems of Intensive Development of Animal Husbandry, 15(1), 358-364 (in Russian).

Lapage, G. (1961). A list of the parasitic protozoa, helminths and arthropoda recorded from species of the family Anatidae (ducks, geese and swans). Parasitology, $51,1-109$.

Marinova, M. H., Boyko, B. G., \& Gergana, P. V. (2013). A checklist of cestodes (Platyhelminthes: Cestoda) of waterfowl (Aves: Anseriformes) in Bulgaria. Acta Zoologica Bulgarica, 65(4), 537-546.

McDonald, M. E. (1969). Catalogue of helminths of waterfowl (Anatidae). United States National Government Publication, Washington.

Moghe, M. A. (1933). Four new species of avian cestodes from India. Parasitology, 25(3), 333-341.

Muhairwa, A. P., Msoffe, P. L., Ranadhnani, S., Mollel, E. L., Mtambo, M. M. A., \& Kassuku, A. A. (2007). Prevalence of gastro-intestinal helminths in free range ducks in Morogoro Municipality, Tanzania. Livestock Research for Rural Development, 19(48), 1-5.

Ola-Fadunsin, S. D., Ganiyu, I. A., Rabiu, M., Hussain, K., Sanda, I. M., Musa, S. A., Uwabujo, P. I., \& Furo, N. A. (2016). Gastrointestinal parasites of different avian species in Ilorin, North Central Nigeria. Journal of Advanced Veterinary and Animal Research, 6(1), 108-116.

Olson, P. D., Littlewood, D. T., Bray, R. A., \& Mariaux, J. (2001). Interrelationships and evolution of the tapeworms (Platyhelminthes: Cestoda). Molecular Phylogenetics and Evolution, 19(3), 443-467.

Owen, R. W. (1951). The helminth parasites of domesticated birds in Mid Wales. Joumal of Helminthology, 25, 105-130.

Paul, B. T., Lawal, J. R., Ejeh, E. F., Ndahi, J. J., Peter, I. D., Bello, A. M., \& Wakil, Y. (2015). Survey of helminth parasites of free range Muscovy ducks (Anas platyrynchos) slaughtered in Gombe, North Eastern Nigeria. International Journal of Poultry Science, 14(8), 466-470.

Petrochenko, V. I., \& Kotelnikov, G. A. (1976). Gel'mintozy ptits [Helminthiasis of birds]. Kolos, Moscow (in Russian)

Ransom, B. H. (1909). The taenioid cestodes of North American birds. Bulletin of the United States National Museum, 69, 1-141.

Rukambile, E. J., Chengula, A., Swai, E. S., \& Jongejan, F. (2020). Poultry ecto-, endo- and haemoparasites in Tanzania: A review. Austin Joumal of Veterinary Science and Animal Husbandry, 7(1), 1066.

Ryzhikov, K. M. (1967). Opredelitel' gel'mintov domashnikh vodoplavayushchikh ptits [Keys to helminths of domestic waterfowl]. Nauka, Moscow (in Russian).

Ryzhikov, K. M., Gubanov, N. M., Tolkacheva, L. M., Khokhlova, I. G., Zinovyeva, E. N., \& Sergeeva, T. P. (1974). Gel'minty ptits Yakutii i sopredel'nykh territoriy. Tsestody i trematody [Helminths of birds of Yakutia and adjacent territories. Cestodes and Trematodes]. Nauka, Moscow (in Russian).

Rzayev, F. H. (2013). Sravnitel'naya gel'mintofauna domashnikh vodoplavayushchikh ptits (Anser anser dom. i Anas platyrhynchos dom.) v razlichnykh ekologicheskikh zonakh Azerbaydzhana [Comparative helminth fauna of domestic waterfowl (Anser anser dom. and Anas platyrhynchos dom.) in various ecologi- cal zones of Azerbaijan]. In: Proceedings of young scientists of the Volga region. Cassandra, Tolyatti. Pp. 145-151 (in Russian).

Rzayev, F. H., \& Ibrahimova, N. E. (2015). Sravnitel'naya kharakteristika gel'mintofauny domashnikh vodoplavayushchikh ptits yugo-vostochnoy chasti Azerbaydzhana [Comparative characteristics of helminthofauna of domestic water birds of the Southeast of Azerbaijan]. Ecological Bulletin, 32, 101-106 (in Russian).

Rzayev, F. H., Seyidbeyli, M. I., Maharramov, S. H., \& Gasimov, E. K. (2020). Formy i ultrastrukturnye osobennosti lateral'nykh kryl'ev gel'minta Trichostrongylus tenuis Mehlis, 1846 (Nematoda: Trichostrongylidae) [Forms and ultrastructural features of the lateral alae of the helminth Trichostrongylus tenuis Mehlis, 1846 (Nematoda: Trichostrongylidae)]. The Journal of V. N. Karazin Kharkiv National University, 34, 112-119 (in Russian).

Schmelz, O. (1941). Quelques cestodes nouveaux d'oiseaux d'Asie [Some new cestodes from Asian birds]. Swiss Journal of Zoology, 48(3), 143-199 (in France).

Seyidbeyli, M. I., \& Maharramov, S. H. (2018). Gel'mintofauna domashnikh vodoplavayushchikh ptits (gus' - Anser anser dom. i utka - Anas platyrhynchos dom.) Nakhchyvanskoy AR [Helminth fauna of domestic waterfowl (goose Anser anser dom. and duck - Anas platyrhynchos dom.) of Nakhchivan AR]. The Joumal of V. N. Karazin Kharkiv National University, 31, 107-113 (in Russian).

Seyidbeyli, M. I., \& Rzayev, F. H. (2018). Helminth fauna of waterfowl poultry in the territory of Babek region of Nakhcivan AR. Journal of Entomology and Zoology Studies, 6(1), 1668-1671.

Seyidbeyli, M. I., Rzayev, F. H., \& Gasimov, E. K. (2020). Ul'trastrukturnye osobennosti kozhno-muskul'nogo meshka gel'minta Trichostrongylus tenuis (Mehlis, 1846) (Nematoda: Trichostrongylidae) [Ultrastructural features of the body wall of the helminth Trichostrongylus tenuis (Mehlis, 1846) (Nematoda: Trichostrongylidae)]. Parasitologiia, 54(5), 402-412 (in Russian).

Shakhtakhtinskaya, Z. M. (1952). Gel'mintofauna okhotnich'e-promyslovykh ptits Azerbaydzhanskoy SSR [Helminthofauna of hunting and game birds in AzSSR]. Elm, Baku (in Russian).

Shakhtakhtinskaya, Z. M. (1959). Gel'minty domashnikh i okhotnich'e-promyslovykh vodoplavayushchikh ptits v Azerbaydzhanskoy SSR [Helminths of domestic and hunting waterfowl in AzSSR]. Works on helminthology for the 80th anniversary of academician K. N. Scrjabin. Nauka, Moscow. Pp. 197-202 (in Russian).

Sharma, K. N. (1943). On some helminths from Burmese ducks (Anas boschas) new to science. Indian Veterinary Joumal, 19(5), 227-232.

Shirinov, N. M. (1961). Gel'mintofauna i gel'mintozy domashnikh vodoplavayushchikh ptits Azerbaydzhanskoy SSR i ispytaniye piperazin-sul'fata pri ganguleterakidoze [Helminth fauna and helminthiasis of domestic waterfowl of the Azerbaijan SSR and the test of piperazine sulfate in ganguleteracidosis]. Elm, Baku (in Russian)

Smogorzhevskaya, L. A. (1976). Gel'minty vodoplavayushchikh i bolotnych ptits fauny Ukrainy [Helminths of waterfowl and wading birds of the fauna of Ukraine]. Nauka Dumka, Kiev (in Russian).

Soliman, K. N. (1955). Observations on some helminth parasites from ducks in Southem England. Journal of Helminthology, 29, 17-26.

Southwell, T. (1930). The fauna of British India: Including Ceylon and Burma. Cestoda. Nature, 126, 951 .

Spasskaya, L. P. (1966). Tsestody ptits SSSR, Gimenolepididy [Cestodes of birds of the USSR, Hymenolepidids]. Nauka, Moscow (in Russian).

Spassky, A. A. (1963). Osnovy tsestodologii. Gimenolepididy-lentochnye gel'minty dikikh i domashnikh ptits [Fundamentals of cestodology. Hymenolepidids - tapeworms of wild and domestic birds]. Nauka, Moscow (in Russian).

Spassky, A. A. (1965). Tsestody roda Aploparaksis ot ptits Kamchatki [Cestodes of the genus Aploparaksis from birds of Kamchatka]. In: Parasitic worms of domestic and wild animals. Academy of Science USSR, Vladivostok. Pp. 303 311 (in Russian).

Sugimoto, M. (1934). Morphological studies on the avian cestodes from Formosa. Joumal of the Central Society for Veterinary Medicine, 47(9), 697-749.

Sultanov, M. A. (1963). Gel'minty domashnikh i okhotnich'e-promyslovykh ptits Uzbekistana [Helminths of domestic and game birds of Uzbekistan]. Fan, Tashkent (in Russian).

Tolkacheva, L. M. (1971). K izucheniyu fauny tsestod gusinykh ptits Yakutii [To the study of the fauna of cestodes of goose birds of Yakutia]. In: Harmful insects and helminths of Yakutia. Yakutknigoizdat, Yakutsk. Pp. 102-108 (in Russian).

Uchida, A., Uchida, K., Itagaki, H., \& Kamegai, S. (1991). Check list of helminth parasites of Japanese birds. Japanese Joumal of Parasitology, 40(1), 7-85.

Vahidova, S. M. (1978). Gel'minty ptits Azerbaydzhana [Helminths of birds of Azerbaijan]. Elm, Baku (in Russian).

Vahidova, S. M., Shirinov, N. M., \& Samedov, N. A. (1982). Atlas, Azerbaycanda ev gushlarinin esas helmintozlari [Atlas, main helminthiasis of poultry in Azerbaijan]. Elm, Baku (in Azerbaijanian).

Yamaguti, S., \& Mitunaga, Y. (1943). Trematodes of birds from Formosa. Transactions, Natural History Society of Taiwan (Formosa), 33(241), 312-329. 
Yevstafyeva, V. A., \& Yeresko, V. I. (2018). Associative course of capillariasis geese in the Poltava region. Scientific Messenger of Lviv National University of Veterinary Medicine and Biotechnologies, 20(83), 73-76.

Yevstafyeva, V., Melnychuk, V., Yeresko, V., Lukyanova, G., \& Gurenko, I. (2018) Osobennosti vidovogo sostava i kharakter raspredeleniya gel'mintov v populyatsii domashnego gusya (Anser anser dom.) [Species composition and distribution of helminths in domestic goose (Anser anser dom.) population]. Veterinary Medicine, 10,34-39 (in Russian).

Yevstafyeva, V., Yeresko, V., Melnychuk, V., \& Bakhur, T. (2020). Prevalence and co-infection of Baruscapillaria genus (Nematoda, Capillariidae) in domestic geese in Ukraine. Folia Veterinaria, 64(1), 32-38.
Yousuf, M. A., Das, P. M., \& Banowary, A. B. (2009). Gastro-intestinal helminths of ducks: Some epidemiologic and pathologic aspects. Journal of the Bangladesh Agricultural University, 7(1), 91-97.

Yuskiv, I. D., \& Melnychuk, V. (2020). Spetsial'ni zaxodi protigel'mintoznogo kompleksu za nayavnosti endoparazitoziv u gusey (Anser anser domesticus \& Anser cygnoides domesticus Linnaeus, 1758) [Special measures of anti-helmintoses complex in case of goose endoparasitoses (Anser anser domesticus \& Anser cygnoides domesticus Linnaeus, 1758)]. Bulletin of Poltava State Agrarian Academy, 1, 222-242 (in Ukrainian). 